\title{
NFIB promotes the progression of gastric cancer by upregulating circMAP7D1 to stabilize HER2 mRNA
}

\author{
HUIMIN YANG ${ }^{1}$, ZHENGZHEN WU $^{2}$, XIN LIU $^{1}$, MIN CHEN $^{3}$, XIN ZHANG $^{3}$ and YONG JIANG ${ }^{3}$ \\ ${ }^{1}$ Department of Pathology, School of Basic Medical Science, North Sichuan Medical College; \\ ${ }^{2}$ Department of Emergency Medicine, The Second Clinical Medical College of North Sichuan Medical College and \\ Nanchong Central Hospital, Nanchong, Sichuan 637000; ${ }^{3}$ Department of Radiation Oncology, \\ Chongqing University Cancer Hospital and Chongqing Cancer Institute and \\ Chongqing Cancer Hospital, Chongqing 400030, P.R. China
}

Received July 21, 2020; Accepted January 4, 2021

DOI: $10.3892 / \mathrm{mmr} .2021 .11908$

\begin{abstract}
The present study evaluated the expression levels of nuclear factor I B (NFIB) in gastric cancer (GC) specimens and cells, and its regulatory roles were further elucidated. The expression levels of NFIB were examined in GC and paired normal specimens, and in human GC and normal gastric epithelial cells by reverse transcription-quantitative PCR. A circular RNA (circRNA) microarray was performed to identify the novel downstream circRNA of NFIB. Cell proliferation was determined by Cell Counting Kit- 8 assay. Furthermore, cell cycle distribution and apoptosis were assessed using flow cytometry. Interactions between RNA were examined by RNA pulldown assay and the stability of target mRNA was evaluated using a mRNA stability assay. The results of the present study revealed that NFIB was upregulated in GC. Furthermore, silencing NFIB suppressed the proliferation of GC cells, whereas cell cycle arrest and apoptosis were enhanced. In addition, significant downregulation of circMAP7D1 (hsa_circ_0004093) was observed in GC cells infected with short hairpin RNA-NFIB. These findings indicated that circMAP7D1 may be a promising downstream molecule of NFIB in GC, and further functional analyses indicated that circMAP7D1 was involved in NFIB-modulated GC cell proliferation and apoptosis. Moreover, human epidermal growth factor receptor 2 (HER2) was identified as a novel target of circMAP7D1 in GC, and NFIB was able to increase the stability of HER 2 mRNA through regulating circMAP7D1. In conclusion, the present findings indicated that NFIB expression was increased in GC. In addition, NFIB may
\end{abstract}

Correspondence to: Dr Yong Jiang, Department of Radiation Oncology, Chongqing University Cancer Hospital and Chongqing Cancer Institute and Chongqing Cancer Hospital, 181 Hanyu Road, Chongqing 400030, P.R. China

E-mail: jiangyongcqch@163.com

Key words: nuclear factor I B, circMAP7D1, human epidermal growth factor receptor 2, gastric cancer, apoptosis promote the proliferation of GC cells and function through stabilizing HER2 mRNA by upregulating circMAP7D1. Notably, NFIB and its novel downstream signaling pathway may serve essential roles during the development of GC, and NFIB may be considered a promising candidate for the treatment of patients with GC.

\section{Introduction}

Gastric cancer (GC) is considered one of the leading causes of cancer-associated mortality and the global incidence of GC has risen in recent years (1). The onset and progression of GC is complex, and the underlying mechanisms remain largely unknown (2). Furthermore, the overall survival rate of patients with $\mathrm{GC}$ is poor $(1,2)$. Although therapeutic approaches for $\mathrm{GC}$ have improved due to the broad usage of endoscopic/surgical resection, the prognosis of GC remains unfavorable $(3,4)$. The therapeutic outcome of patients with GC is closely associated with disease stage at diagnosis, and the mortality rate of patients with advanced GC is high (3-5). Therefore, it is important to discover novel diagnostic/prognostic biomarkers for $\mathrm{GC}$; therefore, the treatment for this disease can be improved.

The nuclear factor I B (NFIB) gene functions as a transcription factor (6). NFIB serves essential roles in embryonic development and tissue differentiation in the fetus (6). NFIB is widely expressed in embryonic/adult tissues, and is abundantly detected in the lungs, skeletal muscle and heart (6). Upregulation of NFIB has been observed in numerous types of cancer, such as GC, breast, lung and prostate cancer, and it is considered a common oncogene (6-9). Furthermore, Wu et al (9) revealed that NFIB was able to promote GC cell growth and metastasis by targeting Akt/Stat3 signaling, which could be a promising molecular mechanism for the treatment of patients with GC. Circular RNAs (circRNAs) are a novel class of non-coding RNAs, which form a continuous loop and have a more stable structure than their linear counterparts (10). The roles of circRNAs have been widely investigated. It has been revealed that certain circRNAs function as potential gene regulators; however, the detailed mechanisms of most circRNAs remain largely unknown (10). circRNAs are involved in the pathogenesis of various diseases, including nervous system disorders 
and cancer (11-14). Furthermore, circSFMBT2 and circ100269 have been reported to be involved in the proliferation of GC cells, consequently affecting tumor progression in GC $(13,14)$. Therefore, circRNAs may serve essential roles during the development of GC; however, the detailed regulatory roles of circRNAs in $\mathrm{GC}$ remain elusive and require further investigation. Human epidermal growth factor receptor 2 (HER2) is a key regulator during the development of breast cancer (15). Previous studies have indicated that HER2 overexpression may be a frequent abnormality in numerous types of cancer, including GC (15-17). HER2 molecular targeted therapy has been introduced for patients with advanced GC, and HER2 status could be essential to select patients who may benefit from targeted therapy (17).

In the present study, the expression levels of NFIB were evaluated in GC specimens and cells, and its regulatory roles were further elucidated. Our data suggested that NFIB could be considered a promising target for the precise treatment of patients with GC.

\section{Materials and methods}

Clinical specimens. A total of 50 GC (29 men and 21 women; age range, $36-67$ years; mean age, $53 \pm 14.2$ years) tissues and matched para-carcinoma samples ( $\geq 5 \mathrm{~cm}$ from tumor margin) were obtained at the Affiliated Hospital of North Sichuan Medical College (Nanchong, China) between August 2010 and September 2015. The exclusion criteria were as follows: Patients that received radiotherapy/chemotherapy prior to surgery were excluded. Immediately upon collection, tissues were sectioned into slices, snap-frozen in liquid nitrogen and stored at $-80^{\circ} \mathrm{C}$ until further use. Patient biopsies were examined by two independent pathologists and all samples were anonymized. The experimental protocol was approved by the Medical Ethics Committee of the Affiliated Hospital of North Sichuan Medical College. Written informed consent was obtained from all patients. Upregulation of NFIB was also revealed by Gene Expression Profiling Interactive Analysis (GEPIA; http://gepia.cancer-pku.cn/).

Cell culture. Human GC cell lines AGS and MKN-45, and the normal human gastric epithelial cell line GES-1 were purchased from the American Type Culture Collection. The cells were cultured in DMEM containing $10 \% \mathrm{FBS}, 100 \mu \mathrm{g} / \mathrm{ml}$ streptomycin and $100 \mathrm{U} / \mathrm{ml}$ penicillin (all from Cytiva). The cells were maintained at $37^{\circ} \mathrm{C}$ in a humidified incubator containing $5 \% \mathrm{CO}_{2}$.

Infection and transfection. To generate the knockdown cell models of NFIB and circMAPK7D1, short hairpin RNAs (shRNAs) targeting NFIB (sh-NFIB) and circMAPK7D1 (sh-circMAPK7D1), and a negative control (sh-NC) were purchased from Shanghai Genepharma Co., Ltd. After annealing, shRNA fragments were integrated into lentiviral pU6-Luc-Puro vector (Shanghai Genepharma Co., Ltd.). Cells $\left(4 \times 10^{5}\right)$ were infected with shRNA lentiviruses (MOI, 50) and incubated at $37^{\circ} \mathrm{C}$ for $24 \mathrm{~h}$. To generate a cell model overexpressing circMAPK7D1, wild-type (oe-circMAPK7D1) or non-targeting (oe-NC) fragments were amplified using PCR, then inserted into PLCDH-cir vector (Invitrogen; Thermo
Fisher Scientific, Inc.). Cells $\left(4 \times 10^{5}\right)$ were transfected with vectors $(1 \mu \mathrm{g})$ at $37^{\circ} \mathrm{C}$ for $48 \mathrm{~h}$. Up- or downregulation of the corresponding genes was confirmed using reverse transcription-quantitative PCR (RT-qPCR). All transfections were conducted using Lipofectamine ${ }^{\circledR} 2000$ (Invitrogen; Thermo Fisher Scientific, Inc.). A total of $12 \mathrm{~h}$ post-transfection, the culture supernatant was replaced with fresh DMEM containing $10 \%$ FBS.

$R T-q P C R$. Total RNA was isolated from clinical specimens or cells using TRIzol $^{\circledR}$ reagent (Invitrogen; Thermo Fisher Scientific, Inc.). Extracted RNA was reverse transcribed into cDNA using PrimeScript ${ }^{\mathrm{TM}}$ RT kit (Takara Biotechnology Co., Ltd.). For RT, the sample was incubated at room temperature for $30 \mathrm{~min}$; subsequently, the sample was incubated at $42^{\circ} \mathrm{C}$ for $45 \mathrm{~min}, 99^{\circ} \mathrm{C}$ for $5 \mathrm{~min}$ and $5^{\circ} \mathrm{C}$ for $5 \mathrm{~min}$ in a PCR cycler. qPCR was carried out using SYBR Green PCR Master Mix (Takara Biotechnology Co., Ltd.), and the reaction was performed on an ABI 7500 Real-Time PCR system (Applied Biosystems; Thermo Fisher Scientific, Inc.) Endogenous GAPDH was used as a reference gene. The sequences used for qPCR were as follows: NFIB, forward, 5'-CCTCACTGGTAC TGGGGTAT-3' and reverse, 5'-TGGACATTGGCCGGTAAG AT-3'; circMAPK7D1, forward, 5'-GCGTCGGTGACTAAG CAATC-3' and reverse, 5'-TCAAGAACAGATGGAAGAATG G-3'; HER2, forward, 5'-GGTCCTGGAAGCCACAAGG-3' and reverse, 5'-GGTTTTCCCACCACATCCTCT-3'; GAPDH, forward, 5'-GTCTCCTCTGACTTCAACAGCG-3' and reverse, 5'-ACCACCCTGTTGCTGTAGCCAA-3'. The PCR program used was as follows: $95^{\circ} \mathrm{C}$ for $5 \mathrm{~min}$, followed by 45 cycles at $95^{\circ} \mathrm{C}$ for $15 \mathrm{sec}, 60^{\circ} \mathrm{C}$ for $20 \mathrm{sec}$ and $72^{\circ} \mathrm{C}$ for $10 \mathrm{sec}$, and a final extension step at $72^{\circ} \mathrm{C}$ for $10 \mathrm{~min}$. Relative gene expression levels were analyzed using the $2^{-\Delta \Delta C \mathrm{C}}$ method (18).

Western blot analysis. Total protein was isolated from tissues or cells using RIPA buffer (Beyotime Institute of Biotechnology). The concentration of the extracted protein was then evaluated using the BCA assay (Beyotime Institute of Biotechnology). Equal amounts of protein $(40 \mu \mathrm{g})$ were separated by SDS-PAGE on $10 \%$ gels and were then transferred onto nitrocellulose membranes (EMD Millipore). Membranes were blocked with Tris-buffered saline (TBS) containing 5\% skimmed milk at room temperature for $1 \mathrm{~h}$, and were incubated with the following primary antibodies: NFIB (1:1,000; cat. no. ab186738; Abcam), HER2 (1:2,000; cat. no. 2242; Cell Signaling Technology, Inc.) or GAPDH (1:2,000; cat. no. sc-32233; Santa Cruz Biotechnology, Inc.) at $4^{\circ} \mathrm{C}$ overnight. Subsequently, the membranes were incubated with HRP-conjugated anti-mouse (1:10,000; cat. no. sc-2371; Santa Cruz Biotechnology, Inc.) or anti-rabbit IgG (1:10,000; cat. no. sc-2357; Santa Cruz Biotechnology, Inc.) at room temperature for $1 \mathrm{~h}$. Protein bands were visualized using ECL detection kit (Pierce; Thermo Fisher Scientific, Inc.) and the blots were semi-quantified using ImageJ software (version 1.48; National Institutes of Health).

Cell Counting Kit-8 (CCK-8) assay. Transfected and infected cells were seeded into a 96-well plate at a density of $3 \times 10^{4}$ cells/well. Subsequently, the proliferative activity of cells was determined on days 1,2,3 and 4. Briefly, $10 \mu \mathrm{l}$ CCK-8 
solution (Beyotime Institute of Biotechnology) was added to each well. After incubation for $2 \mathrm{~h}$ at $37^{\circ} \mathrm{C}$, the absorbance was detected at $450 \mathrm{~nm}$ using a microplate reader (Bio-Rad Laboratories, Inc.).

Cell cycle distribution and apoptosis assay. Transfected and infected cells were seeded into a 6-well plate at a density of $6 \times 10^{5}$ cells/well. Cells were then collected through low-speed centrifugation $(2,600 \mathrm{x} \mathrm{g})$ at $4^{\circ} \mathrm{C}$ for $5 \mathrm{~min}$. Cell pellets were washed and resuspended using PBS, then fixed using pre-chilled ethanol (70\%) at room temperature for $15 \mathrm{~min}$ and stored at $4^{\circ} \mathrm{C}$ overnight. Cells were centrifuged $(10,000 \mathrm{x} \mathrm{g})$ and then resuspended in propidium iodide (PI; Sigma-Aldrich; Merck KGaA) staining buffer with $50 \mu \mathrm{l} / \mathrm{ml}$ PI and $250 \mu \mathrm{l} / \mathrm{ml}$ RNase $\mathrm{A}$ at $4^{\circ} \mathrm{C}$ for $1 \mathrm{~h}$. Cell cycle distribution was evaluated using a flow cytometer (FACSCalibur; BD Biosciences) and was analyzed using FlowJo software (version 7.6; FlowJo LLC). In order to examine cell apoptosis, the cell suspension was incubated with $5 \mu \mathrm{l}$ Annexin V-FITC and $10 \mu \mathrm{l}$ PI solution at $4^{\circ} \mathrm{C}$ in the dark for $30 \mathrm{~min}$ (Shenzhen Jingmei Biotech Engineering Co. Ltd.), and cell apoptotic rate was analyzed by flow cytometry (FACSCalibur; BD Biosciences) and interpreted using FlowJo software. Both early $\left(\mathrm{FITC}^{+} \mathrm{PI}^{-}\right)$and late $\left(\mathrm{FITC}^{+} \mathrm{PI}^{+}\right)$apoptosis were evaluated.

circRNA microarray. Total RNA was extracted using TRIzol ${ }^{\circledR}$ reagent (Invitrogen; Thermo Fisher Scientific, Inc.) and a RNeasy Mini kit (Qiagen $\mathrm{GmbH}$ ). Fluorescence-labeled targets were produced for circRNA array. Human circRNA array v2 (CapitalBio Technology Co., Ltd.) was designed and genes were mounted onto the chip; target sequences of corresponding circRNAs were obtained from Circbase (http://www.circbase.org/). Labeled targets were then hybridized with extracted RNA samples, which were scanned using the Agilent Microarray Scanner (Agilent Technologies, Inc.). All data were normalized according to Quantile algorithm (19). Arrays were conducted using the protocol supplied by Agilent Technologies, Inc.

RNA pulldown assay. Biotinylated wild-type (WT) or mutant (MUT) NFIB (NFIB-WT-Bio/NFIB-MUT-Bio) and circMAP7D1 (circMAP7D1-WT-Bio/circMAP7D1-MUT-Bio) probes, and a negative control (NC-Bio) probe were generated by Shanghai GenePharma Co., Ltd. Briefly, for RNA pulldown assay, RNA was extracted from cells, as aforementioned. Extracted RNA was labeled with biotinylated probes. Lysates of AGS and MKN-45 cells were prepared using lysis buffer containing $\mathrm{KCl}(10 \mathrm{mM}), \mathrm{MgCl} 2(1.5 \mathrm{mM})$, Tris-Cl pH 7.5 (10 mM), DTT (5 mM) and SUPERase. In $^{\text {TM }}(60 \mathrm{U} / \mathrm{ml}$; Thermo Fisher Scientific, Inc.). Lysates were mixed with pro-labeled RNA in the aforementioned lysis buffer. The binding reaction was incubated at room temperature for $1 \mathrm{~h}$ and labeled with Dynabeads M-280 Streptavidin, and the mixture was rotated overnight at $4^{\circ} \mathrm{C}$ (Thermo Fisher Scientific, Inc.). The beads with immobilized NFIB or circMAP7D1 were treated with $10 \mathrm{mM}$ ethylenediaminetetraacetic acid. TRIzol ${ }^{\circledR}$ was used to extract the bound RNAs, which were further subjected to RT-qPCR, as aforementioned. After pulldown, the crosslinked molecules were identified using mass spectrometry as previously described (20).
Assessment of mRNA stability. Cells were seeded into a 6-well plate $\left(6 \times 10^{5}\right.$ cells/well $)$ and incubated at $37^{\circ} \mathrm{C}$ overnight. The cells were then treated with actinomycin D (ActD; $5 \mu \mathrm{g} / \mathrm{ml}$; MedChemExpress) for different durations (15,30 and $60 \mathrm{~min})$ to inhibit further RNA synthesis at $37^{\circ} \mathrm{C}$. Total RNA was isolated and analyzed using RT-qPCR, as aforementioned. The remaining mRNA levels of HER2 at each time point were normalized to the levels detected at the beginning of the experiment.

Luciferase reporter assay. Fragments containing complementary sequences of NFIB were cloned into a luciferase plasmid [WT-NFIB; OBiO Technology (Shanghai) Corp., Ltd.]. A vector carrying mutant fragments of NFIB was used as a control [MUT-NFIB; OBiO Technology (Shanghai) Corp., Ltd.]. AGS cells $\left(4 \times 10^{5} /\right.$ well) were seeded onto a 6 -well plate co-transfected with $1 \mu \mathrm{g}$ luciferase vectors and oe-circMAP7D1/oe-NC (synthesized by Shanghai Genepharma Co., Ltd.) using Lipofectamine 2000. After 48 h, luciferase activity was determined using dual luciferase reporter system kit (Promega Corporation). Luciferase activity was normalized to Renilla luciferase activity.

Immunohistochemistry (IHC). Immunostaining of HER2 was carried out on paraffin-embedded tissue sections (thickness, $10 \mu \mathrm{m}$ ); tissues were fixed with $4 \%$ paraformaldehyde at $4^{\circ} \mathrm{C}$ overnight before embedding. The sections were dewaxed using xylene, and rehydrated through a graded ethanol series and water. Antigen retrieval was performed in a microwave using $10 \mathrm{mM}$ sodium citrate for $15 \mathrm{~min}$. Tissues were then incubated with FBS at room temperature for $30 \mathrm{~min}$ and with a primary antibody against HER2 (1:100; cat. no. 2242; Cell Signaling Technology, Inc.) in a humidified incubator at $4{ }^{\circ} \mathrm{C}$ overnight. Subsequently, the sections were rinsed in TBS three times and incubated with biotinylated secondary antibody (1:100; cat. no. E0432; Dako; Agilent Technologies, Inc.). Protein expression was then detected using a streptavidin-biotin-peroxidase system (ABC kit; Dako; Agilent Technologies, Inc.). Stained slides were observed using a light microscope (magnification, x200; CX23 model; Olympus Corporation). Staining intensity was semi-quantified using ImageJ software (version 1.46).

Statistical analysis. All data are presented as the mean \pm standard deviation and were analyzed using SPSS software (version 17.0; SPSS, Inc.). All assays were performed in triplicate. The significance of differences was analyzed using one-way analysis of variance (ANOVA) or paired/unpaired Student's t-test. Newman-Keuls test was conducted following ANOVA. The relationship between RNA expression was evaluated by Pearson's correlation analysis. Receiver operating characteristic (ROC) curve analysis was performed to assess the power of NFIB expression in distinguishing between GC and para-carcinoma tissues, patients with and without metastasis, and grade III-IV and I-II GC. P $<0.05$ was considered to indicate a statistically significant difference.

\section{Results}

Expression levels of NFIB are increased in GC specimens and cells. The expression levels of NFIB were examined in 50 pairs of GC and para-carcinoma tissues using RT-qPCR. The 

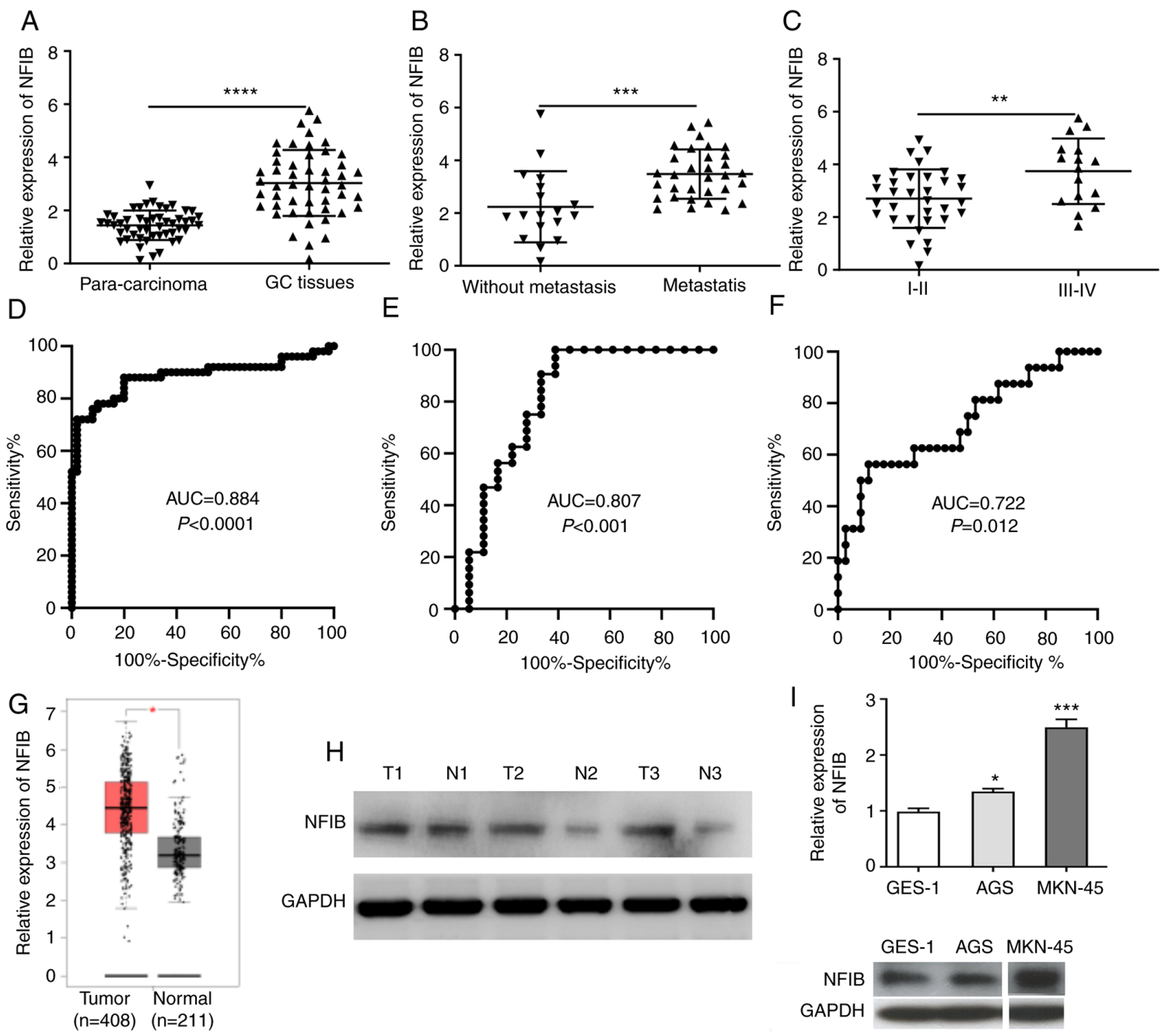

Figure 1. NFIB expression is upregulated in GC tissues and cells. (A) Expression levels of NFIB were examined in 50 GC specimens and matched para-carcinoma tissues by RT-qPCR. (B) NFIB expression was determined in patients with GC with or without metastasis. (C) Expression levels of NFIB were detected in patients with GC at different grades. Receiver operating characteristic curve analyses revealed that the expression profile of NFIB exhibited high AUC values in distinguishing (D) between GC and para-carcinoma samples, (E) between patients with and without metastasis, and (F) between grade III-IV and I-II GC. (G) Upregulation of NFIB was also revealed by Gene Expression Profiling Interactive Analysis. (H) Elevated NFIB expression was observed in GC samples using western blot analysis. (I) RT-qPCR and western blotting indicated that NFIB mRNA and protein expression levels were upregulated in GC cell lines compared with in the normal human gastric epithelial cell line GES-1. Two non-relevant bands were removed from the blots. ${ }^{*} \mathrm{P}<0.05,{ }^{* * *} \mathrm{P}<0.01,{ }^{* * * *} \mathrm{P}<0.001,{ }^{* * * * *} \mathrm{P}<0.0001$ vs. GES-1 or as indicated. AUC, area under curve; GC, gastric cancer; NFIB, nuclear factor I B; RT-qPCR, reverse transcription-quantitative polymerase chain reaction; T, tumor; N, normal.

expression levels of NFIB were significantly elevated in GC specimens compared with those in the normal control specimens (Fig. 1A). Furthermore, the association between NFIB expression and the progression of GC was investigated. The expression levels of NFIB were significantly upregulated in patients with GC and metastasis compared with those in patients with GC without metastasis (Fig. 1B). In addition, the expression of NFIB was significantly increased in patients with grade III-IV GC compared with that in patients with grade I-II GC (Fig. 1C). Moreover, ROC curve analyses suggested that NFIB expression signature exhibited a high area under the curve value in distinguishing between GC and para-carcinoma tissues, between GC with and without metastasis, and between grade III-IV GC and I-II GC (Fig. 1D-F). Consistent with the present findings, upregulation of NFIB was also revealed by Gene Expression Profiling Interactive Analysis (GEPIA; http://gepia.cancer-pku. $\mathrm{cn} /$ ) (Fig. 1G). Furthermore, increased protein expression levels of NFIB were detected in GC specimens compared with those in normal control specimens, as determined using western blotting (Fig. 1H). In addition, the mRNA and protein expression levels of NFIB were upregulated in GC cell lines compared with those in normal human gastric epithelial cells (Fig. 1I). These findings indicated that the expression levels of NFIB were elevated in GC and may contribute to tumor development.

Knockdown of NFIB inhibits the proliferation of GC cells and promotes apoptosis. To identify the roles of NFIB in tumor progression of GC, NFIB was knocked down in AGS and MKN-45 cells. Infection efficiency was confirmed by RT-qPCR and western blotting (Fig. 2A). In addition, CCK-8 assay suggested that the proliferation of GC cells infected with sh-NFIB was significantly reduced (Fig. 2B and C). To further investigate the effects of NFIB on GC cell proliferation, cell cycle distribution and apoptosis were examined in GC cells infected with sh-NFIB compared with the control cells. The results revealed that the percentage of cells in $\mathrm{G}_{0} / \mathrm{G}_{1}$ phase was significantly elevated, whereas the percentage of cells in $\mathrm{S}$ phase was notably reduced following NFIB knockdown, suggesting that the GC cell cycle was 

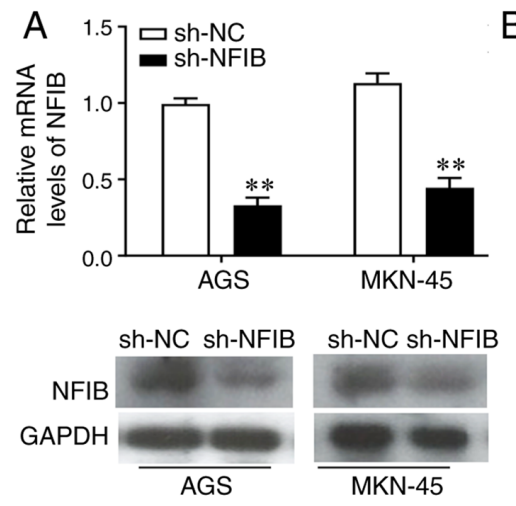

D

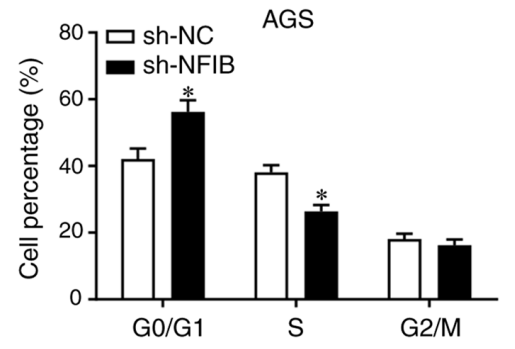

E

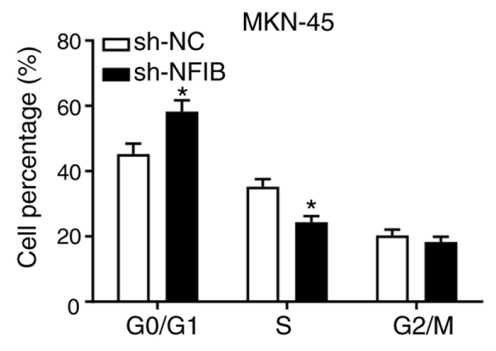

$\mathrm{F}$

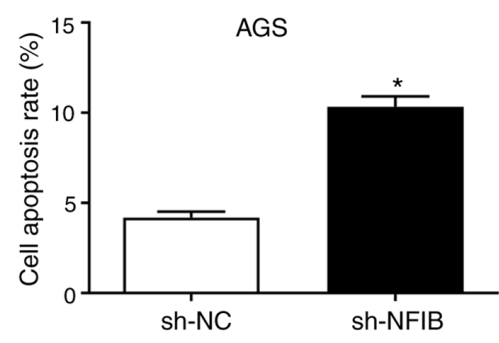

G

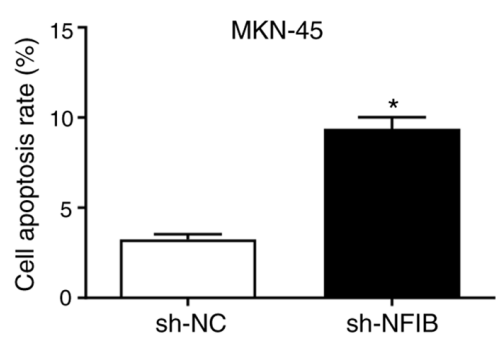

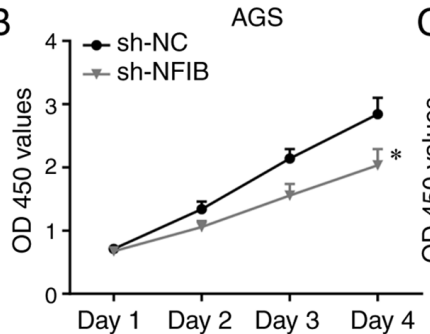
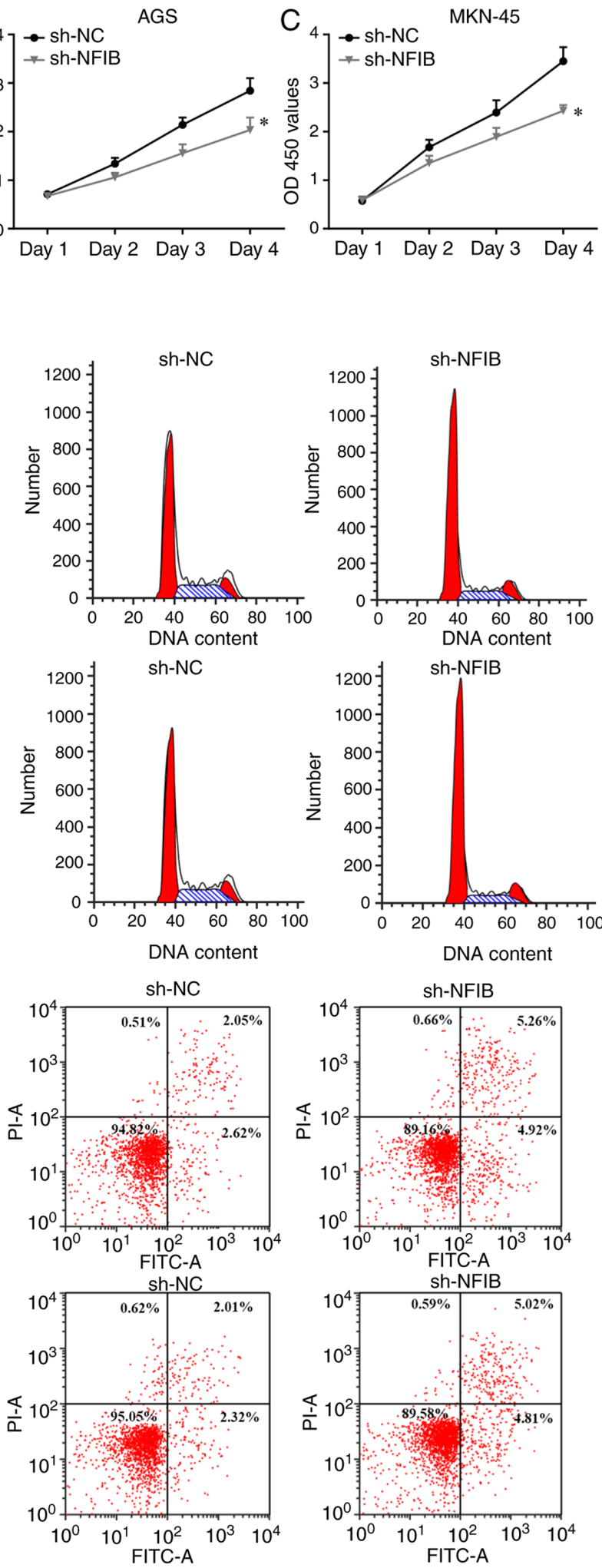

Figure 2. NFIB knockdown suppresses the proliferation and promotes the apoptosis of GC cells. (A) Infection efficiency of sh-NFIB was evaluated by reverse transcription-quantitative polymerase chain reaction. (B and C) Proliferation of GC cells was examined post-infection with sh-NFIB using Cell Counting Kit-8 assay. (D and E) Cell cycle progression of GC cells infected with sh-NFIB was analyzed. (F and G) Apoptotic rates of GC cells were determined post-infection with sh-NFIB. ${ }^{*} \mathrm{P}<0.05,{ }^{* *} \mathrm{P}<0.01$ vs. sh-NC. GC, gastric cancer; NC, negative control; NFIB, nuclear factor I B; OD, optical density; PI, propidium iodide; sh, short hairpin RNA.

shifted from $S$ and $G_{2} / M$ phase to $G_{0} / G_{1}$ phase (Fig. $2 D$ and $E$ ). Moreover, flow cytometry indicated that knockdown of NFIB enhanced the apoptosis of GC cells (Fig. 2F and G). These results indicated that knockdown of NFIB may promote cell cycle arrest in $\mathrm{G}_{0} / \mathrm{G}_{1}$ phase and enhance the apoptosis of $\mathrm{GC}$ cells.
circMAP7D1 is a novel target of NFIB in GC. In order to investigate the mechanisms of NFIB-mediated signaling, the downstream molecules of NFIB were predicted using circRNA microarray. The expression profiles of circRNAs were evaluated in GC cells infected with sh-NC or sh-NFIB. 
The expression levels of circMAPK7D1 were markedly downregulated following NFIB knockdown (Fig. 3A). Furthermore, RT-qPCR revealed the upregulation of circMAP7D1 in GC specimens and cells compared with in the normal controls (Fig. 3B and C). RNA pulldown assay indicated that NFIB was able to interact with circMAP7D1 in GC cells (Fig. 3D). Furthermore, the interaction between NFIB and circMAP7D1 was confirmed using a luciferase assay; overexpressed circMAP7D1 significantly suppressed luciferase activity of the plasmids containing WT-NFIB, but not the MUT control (Fig. 3E), indicating that WT-NFIB could interact with circMAP7D1 in GC cells. In addition, the expression levels of circMAP7D1 were markedly decreased in GC cells infected with sh-NFIB (Fig. 3E), and a positive correlation was observed between NFIB and circMAP7D1 expression in GC tissues (Fig. 3F). These findings suggested that circMAP7D1 may be a promising target of NFIB in GC cells.

circMAP7D1 is involved in the regulation of $G C$ cell proliferation and apoptosis. To further identify the regulatory roles of circMAP7D1 in the development of GC, AGS and MKN-45 cells were transfected with oe-circMARP7D1A and infected with sh-circMAP7D1, and transfection and infection efficiency was confirmed by RT-qPCR (Fig. 3G and H). Notably, cell proliferation was significantly enhanced by oe-circMAP7D1 and suppressed by sh-circMAP7D1 (Fig. 3I and J). Moreover, cell cycle arrest and apoptosis were inhibited post-transfection with oe-circMAP7D1 and were promoted post-infection with sh-circMAP7D1 (Fig. 3K-N). These results revealed that circMAP7D1 may be involved in the regulation of GC cell proliferation and apoptosis.

Stability of HER2 mRNA is enhanced by circMAP7D1. To identify the putative target of circMAP7D1 in GC cells, a further functional study was performed. The results of RNA pulldown assay indicated that circMAP7D1 could interact with HER2 in GC cells (Fig. 4A). Briefly, following pulldown, the crosslinked molecules were identified using mass spectrometry (data not shown), and HER2 was selected for further experiments. Notably, overexpression of circMAP7D1 significantly increased the stability of HER2 mRNA, whereas HER2 stability was markedly decreased by the knockdown of circMAP7D1 (Fig. 4B and C). In consistence with the present results, upregulated levels of HER2 were detected in GC samples by GEPIA (Fig. 4D). In addition, IHC indicated that the protein expression levels of HER2 were markedly increased in GC tissues compared with in para-carcinoma control tissues (Fig. 4E). Furthermore, the expression levels of HER2 were elevated in GC cells transfected with oe-circMAP7D1 and reduced post-infection of cells with sh-circMAP7D1 (Fig. 4F and G). Additionally, the mRNA expression levels of HER2 were markedly decreased in GC cells infected with sh-NFIB (Fig. 4H). These findings indicated that circMAP7D1 may be able to stabilize HER2 mRNA in GC.

circMAP7D1 is involved in NFIB-modulated GC cell proliferation and apoptosis. To further identify the roles of circMAP7D1 in the regulation of GC cell proliferation, a functional analysis was carried out. Western blotting revealed that the protein expression levels of HER2 were reduced in GC cells infected with sh-NFIB, whereas these effects were reversed by overexpression of circMAP7D1 and were strengthened by circMAP7D1 knockdown (Fig. 4I and J). Furthermore, the apoptotic rates of GC cells were significantly increased post-infection with sh-NFIB, whereas this effect was abrogated by oe-circMAP7D1 and enhanced by sh-circMAP7D1 (Fig. 4K and L). These findings suggested that NFIB was able to promote the proliferation of GC cells, and that NFIB exerted its regulatory function by upregulating circMAP7D1, which subsequently stabilized HER 2 mRNA.

\section{Discussion}

NFIB is considered to be an oncogenic factor in tumor progression, and increased levels of NFIB have been detected in malignancies, such as GC (6-9). In the present study, upregulation of NFIB was revealed in GC specimens and cells, and NFIB knockdown markedly suppressed the proliferation of GC cells. Emerging evidence has indicated that circRNAs are associated with tumorigenesis in various types of cancer and dysregulation of circRNAs has also been detected (13,14,21-25). Moreover, previous studies have elucidated the important roles of circRNAs in the pathogenesis of GC (26-28); however, at present, the detailed function of most circRNAs in GC are not completely understood.

The present findings revealed that the expression levels of circMAP7D1 were elevated in GC tissues, and NFIB and circMAP7D1 expression was positively correlated. Moreover, an RNA pulldown assay revealed the interaction between NFIB and circMAP7D1 in GC cells. In addition, the inhibitory effects of sh-NFIB on cell proliferation could be reversed or strengthened by oe-circMAP7D1 or sh-circMAP7D1, respectively. These findings suggested that circMAP7D1 may be a promising target of NFIB in GC and could participate in NFIB-modulated GC cell proliferation.

Recent studies have suggested that upregulation of HER2 could be associated with the development of various types of cancer, including GC (15-17). Numerous oncogenes and growth factors are regulated post-transcriptionally. The key mechanism of post-transcriptional regulation of oncogenes is through controlling the rate of mRNA turnover; until now, only a few regulatory factors have been discovered that could regulate a large pool of target mRNAs, indicating that a minor perturbation during the controlling process could lead to marked effects that may result in the progression of a complex disorder such as cancer (29-31). The present data revealed the interaction between circMAP7D1 and HER2 in GC cells, and HER2 mRNA stability was notably increased by circMAP7D1 overexpression and decreased by circMAP7D1 knockdown. These findings suggested that HER2 could be a novel downstream molecule of circMAP7D1 in GC; moreover, NFIB may stabilize HER2 mRNA through upregulating circMAP7D1. It may be hypothesized that circMAP7D1 could regulate the expression of HER2 through microRNA sponges, or it may affect the expression of HER 2 at the transcriptional or translational level; this hypothesis requires further investigation. However, there were limitations in the present study. For example, cell-counting experiments should be performed to confirm the effects on proliferation. In future, further functional studies should be performed to assess the involvement of circMAP7D1 and HER2 in NFIB-modulated cell cycle progression and apoptosis. 

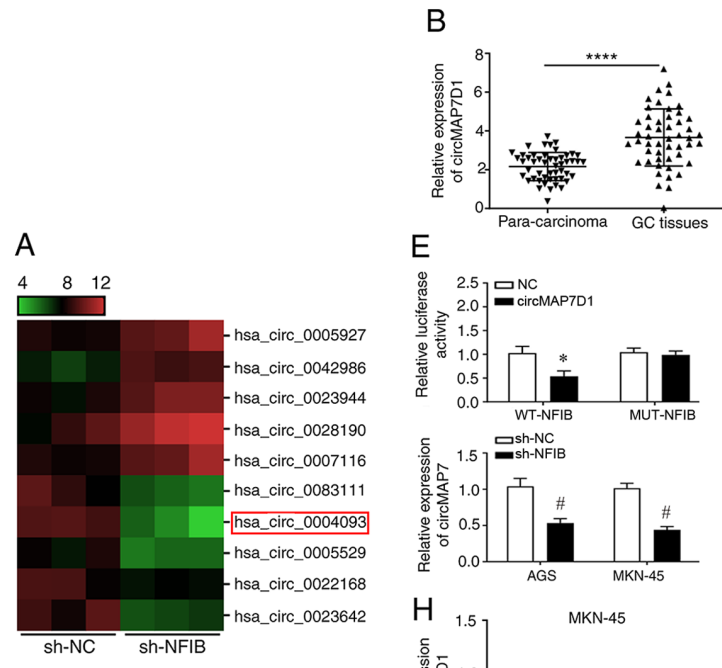

$\mathrm{E}$
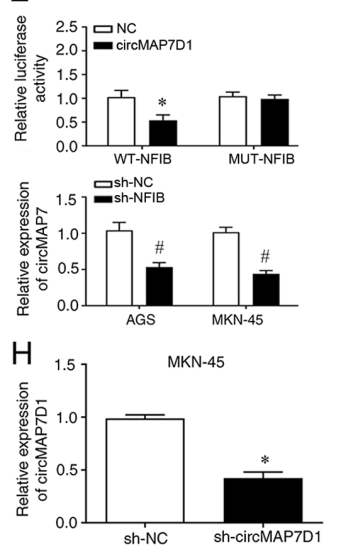
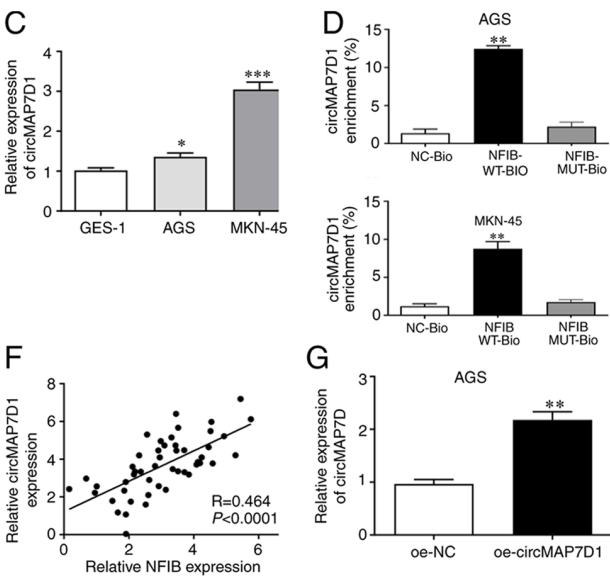

G 3 3GS
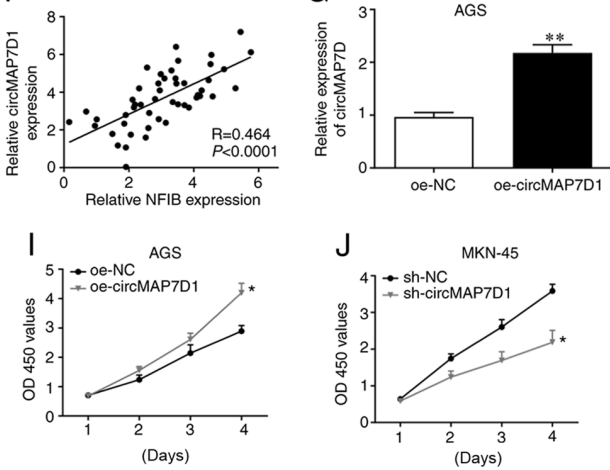
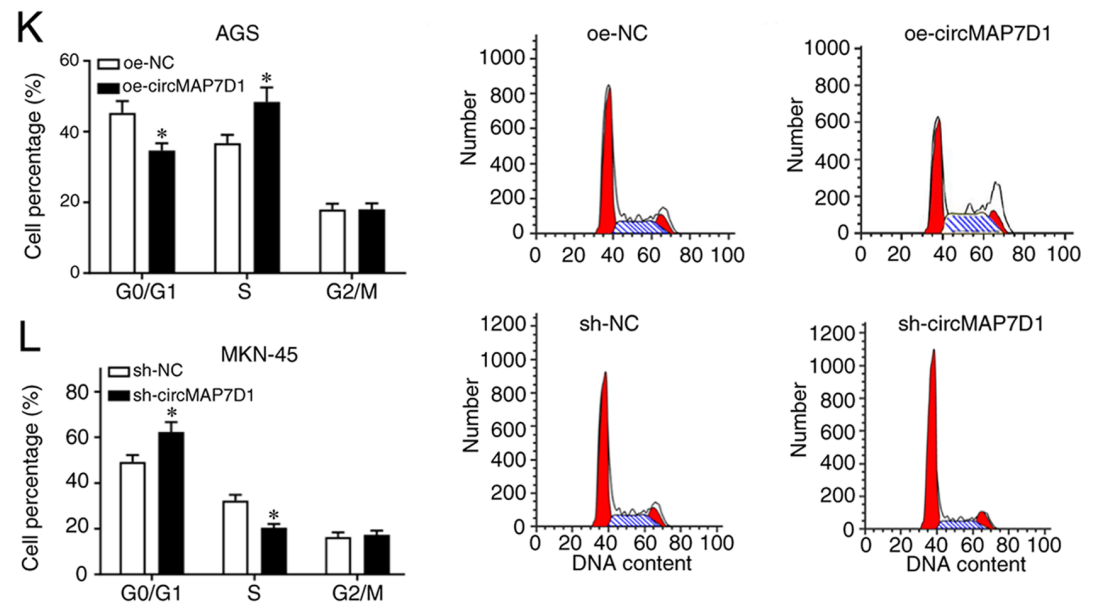

$\mathrm{M}$
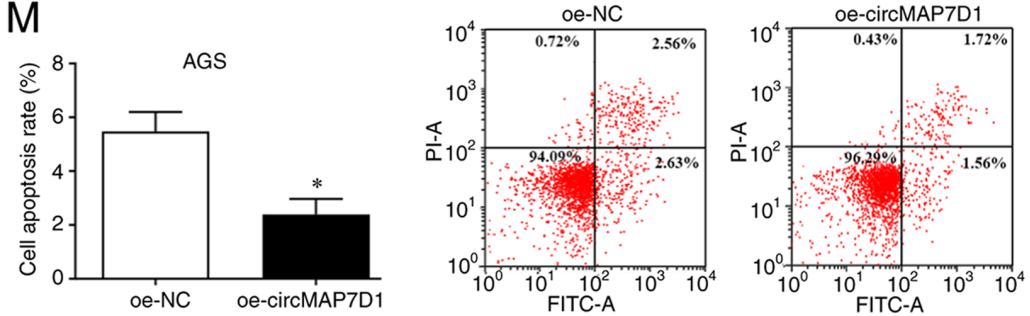

$\mathrm{N}$
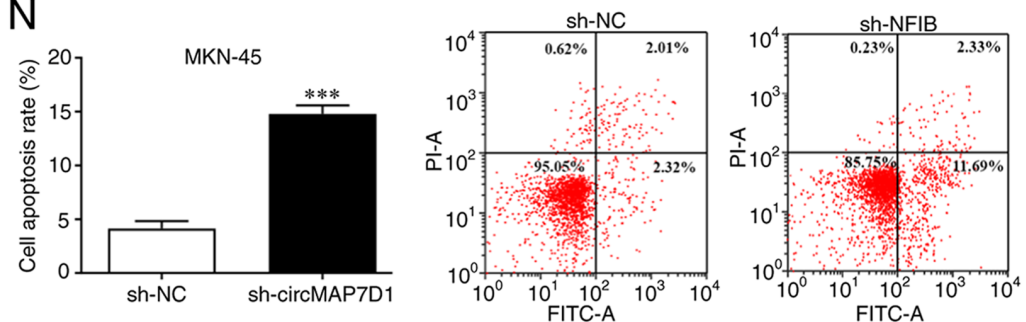

Figure 3. circMAP7D1 is a promising downstream molecule of NFIB in GC and is associated with GC cell proliferation. (A) Expression profiles of circRNAs in GC cells infected with sh-NC and sh-NFIB were evaluated using a circRNA microarray. (B) Expression levels of circMAP7D1 were examined in GC tissues using RT-qPCR. ${ }^{* * * * * *}$ P $<0.0001$ vs. para-carcinoma tissues. (C) Expression levels of circMAP7D1 were detected in cells using RT-qPCR. ${ }^{* P}<0.05$, ${ }^{* * * *} \mathrm{P}<0.001$, vs. GES-1. (D) RNA pulldown assay was conducted to evaluate the interaction between NFIB and circMAP7D1. ${ }^{* *} \mathrm{P}<0.01 \mathrm{vs.}$ NC-Bio. (E) Luciferase activity of WT-NFIB-treated cells was decreased by circMAP7D1, and the expression of circMAP7D1 was reduced in GC cells following the infection with sh-NFIB. ${ }^{~ P}<0.05$ vs. NC, ${ }^{~} \mathrm{P}<0.05$ vs. sh-NC. (F) NFIB and circMAP7D1 expression was positively correlated in GC specimens. (G) GC cells were transfected with oe-circMARP7D1A ${ }^{* *} \mathrm{P}<0.01$ vs. oe-NC. (H) GC cells were infected with sh-circMAP7D1. "P<0.05 vs. sh-NC. (I) Cell proliferation was examined post-transfection with oe-circMARP7D1A. ${ }^{*} \mathrm{P}<0.05$ vs. oe-NC. (J) Cell proliferation was determined post-infection with sh-circMAP7D1. ${ }^{\mathrm{P}}<0.05$ vs. sh-NC. (K) Cell cycle progression and (M) apoptotic rate of GC cells were determined post-transfection with oe-circMARP7D1A. ${ }^{*} \mathrm{P}<0.05$ vs. oe-NC. (L) Cell cycle progression and (N) apoptotic rate of GC cells were evaluated post-infection with sh-circMAP7D1 using flow cytometry. ${ }^{*} \mathrm{P}<0.05,{ }^{* * * *} \mathrm{P}<0.001$ vs. sh-NC. circRNA, circular RNA; GC, gastric cancer; MUT, mutant; NC, negative control; PI, propidium iodide; RT-qPCR, reverse transcription-quantitative polymerase chain reaction; sh, short hairpin RNA; WT, wild-type. 
$\mathbf{A}$

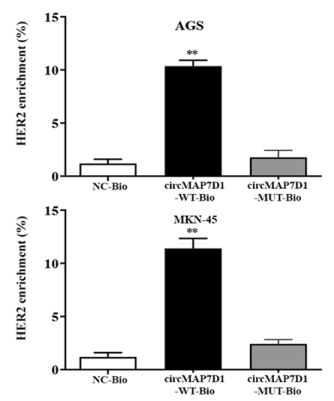

$\mathbf{E}$

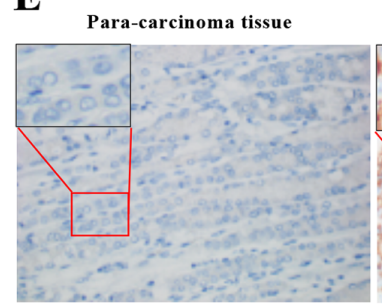

$\mathbf{H}_{2}$

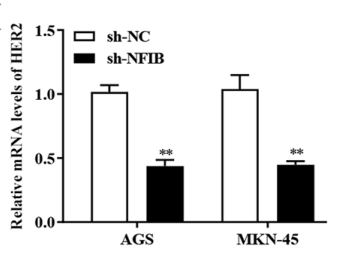

$\mathbf{K}$

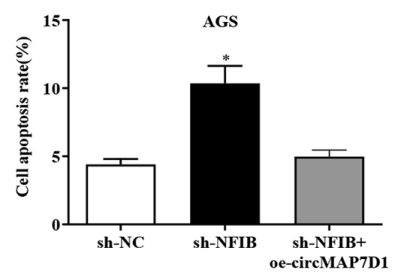

$\mathbf{L}$

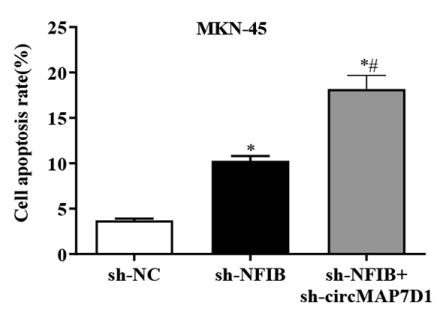

B

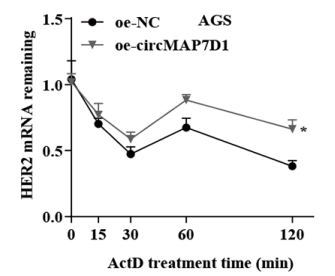

ActD treatment time (min)
C

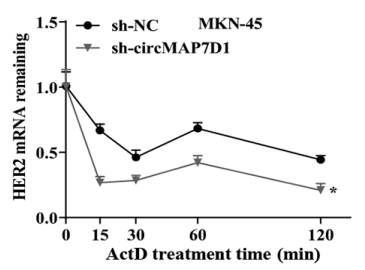

$\mathbf{F}$

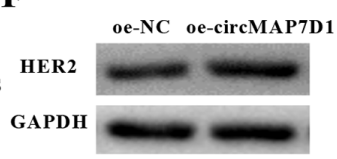

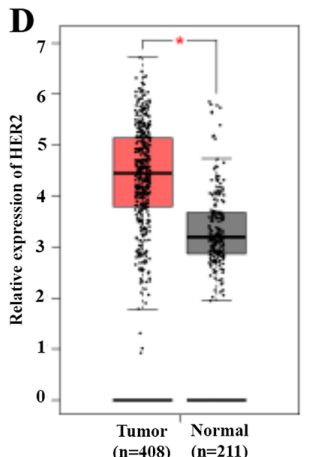

G

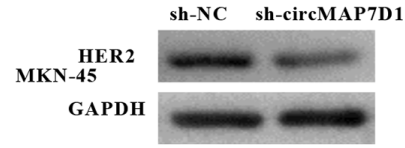

$\mathbf{J}$

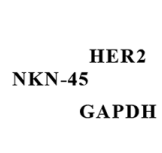

GAPDH
sh-NFIB+

sh-NC sh-NFIB sh-circMAP7D1

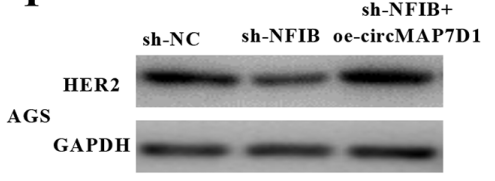

\section{s}

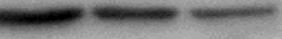

sh-NFIB
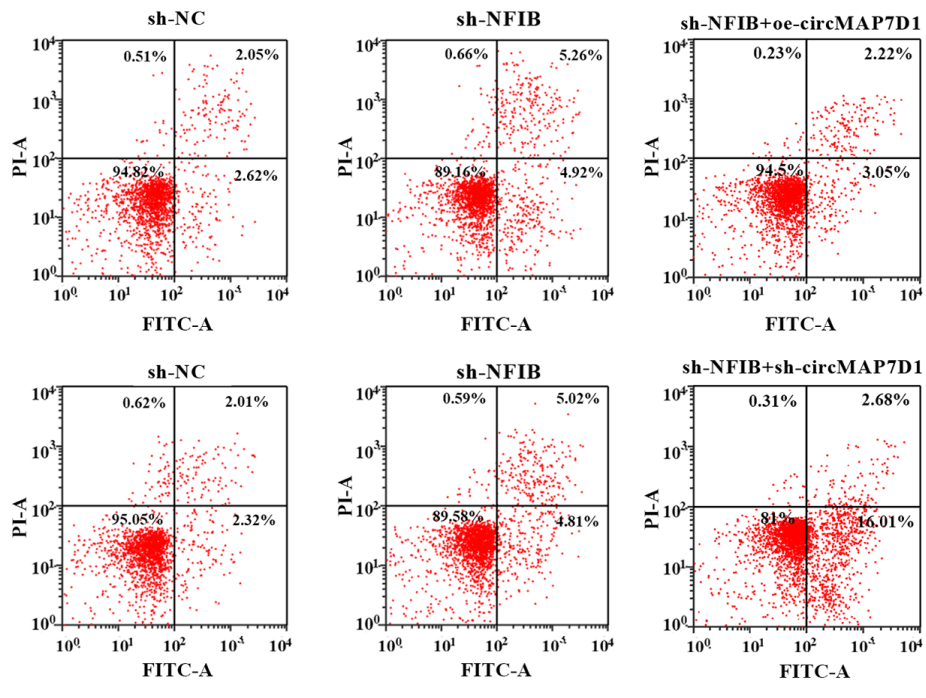

Figure 4. NFIB enhances the stability of HER2 mRNA and affects GC cell proliferation through circMAP7D1. (A) RNA pulldown assay revealed the interaction between circMAP7D1 and HER2 in GC cells. ${ }^{* *} \mathrm{P}<0.01$. vs. NC-Bio. (B) Stability of HER2 mRNA was increased by oe-circMAP7D1. ${ }^{*} \mathrm{P}<0.05$ vs. oe-NC. (C) Stability of HER2 mRNA was reduced by sh-circMAP7D1. "P<0.05 vs. sh-NC. (D) Upregulation of HER2 was revealed by Gene Expression Profiling Interactive Analysis. " $\mathrm{P}<0.05$ vs. normal tissue. (E) Protein expression levels of HER2 were examined in GC samples and normal controls using immunohistochemistry. Expression levels of HER2 were (F) increased by oe-circMAP7D1 and (G) decreased by sh-circMAP7D1 in GC cells. (H) mRNA expression levels of HER2 were reduced in GC cells infected with sh-NFIB. ${ }^{* *} \mathrm{P}<0.01$ vs. sh-NC. (I and J) Protein expression levels of HER2 were evaluated in GC cells in the sh-NFIB or sh-NFIB + oe-circMAP7D1 groups. (K and L) Cell apoptosis was determined in the sh-NFIB or sh-NFIB + oe-circMAP7D1 groups. " $\mathrm{P}<0.05$ vs. sh-NC; ${ }^{\#} \mathrm{P}<0.05$ vs. sh-NFIB. ActD, actinomycin D; GC, gastric cancer; HER2, human epidermal growth factor receptor 2; MUT, mutant; NC, negative control; PI, propidium iodide; sh, short hairpin RNA; WT, wild-type.

In conclusion, the present study provided substantial evidence on the role of NFIB in tumorigenesis and revealed that NFIB was a novel oncogenic factor during the pathogenesis of GC. NFIB could promote the proliferation of GC cells by upregulating circMAP7D1 to stabilize HER2 mRNA, consequently inducing the tumor progression of GC. Therefore, NFIB could be considered a putative candidate for targeted therapy of patients with GC.

\section{Acknowledgements}

Not applicable. 


\section{Funding}

The present study was funded by the Science and Technology Research Project of Chongqing Education Commission (grant no. KJQN201900104).

\section{Availability of data and materials}

The datasets used and/or analyzed during the current study are available from the corresponding author on reasonable request.

\section{Authors' contributions}

YJ designed the present study. HY, ZW, XL, MC and XZ performed the experiments and data analysis. YJ and $\mathrm{HY}$ confirm the authenticity of all the raw data. All authors drafted the manuscript, and read and approved the final manuscript.

\section{Ethics approval and consent to participate}

The experimental protocol was approved by the Medical Ethics Committee of the Affiliated Hospital of North Sichuan Medical College. Written informed consent was obtained from all patients.

\section{Patient consent for publication}

Not applicable.

\section{Competing interests}

The authors declare that they have no competing interests.

\section{References}

1. Lyons K, Le LC, Pham YTH, Borron C, Park JY, Tran CTD, Tran TV, Tran HTT, Vu KT, Do CD, et al: Gastric cancer: Epidemiology, biology, and prevention: A mini review. Eur J Cancer Prev 28: 397-412, 2019.

2. Allemani C, Weir HK, Carreira H, Harewood R, Spika D, Wang XS, Bannon F, Ahn JV, Johnson CJ, Bonaventure A, et al: Globa surveillance of cancer survival 1995-2009: Analysis of individual data for $25,676,887$ patients from 279 population-based registries in 67 countries (CONCORD-2). Lancet 385: 977-1010, 2015.

3. Park YM, Cho E, Kang HY and Kim JM: The effectiveness and safety of endoscopic submucosal dissection compared with endoscopic mucosal resection for early gastric cancer: A systematic review and metaanalysis. Surg Endosc 25: 2666-2677, 2011.

4. Choi MK, Kim GH, Park DY, Song GA, Kim DU, Ryu DY, Lee BE, Cheong JH and Cho M: Long-term outcomes of endoscopic submucosal dissection for early gastric cancer: A single-center experience. Surg Endosc 27: 4250-4258, 2013.

5. Takahashi T, Saikawa Y and Kitagawa Y: Gastric cancer: Current status of diagnosis and treatment. Cancers (Basel) 5: 48-63, 2013.

6. Becker-Santos DD, Lonergan KM, Gronostajski RM and Lam WL: Nuclear factor I/B: A master regulator of cell differentiation with paradoxical roles in cancer. EBioMedicine 22: 2-9, 2017.

7. Dooley AL, Winslow MM, Chiang DY, Banerji S, Stransky N, Dayton TL, Snyder EL, Senna S, Whittaker CA, Bronson RT, et al: Nuclear factor I/B is an oncogene in small cell lung cancer. Genes Dev 25: 1470-1475, 2011.

8. Nanda JS, Awadallah WN, Kohrt SE, Popovics P, Cates JM, Mirosevich J, Clark PE, Giannico GA and Grabowska MM: Nuclear factor I/B increases in prostate cancer to support androgen receptor activation. BioRxiv: Jun 27, 2019 (Epub ahead of print). doi: 10.1002/pros.24019.
9. Wu C, Zhu X, Liu X, Ruan T, Wan W and Tao K: NFIB promotes cell growth, aggressiveness, metastasis and EMT of gastric cancer through the Akt/Stat3 signaling pathway. Oncol Rep 40: 1565-1573, 2018.

10. Memczak S, Jens M, Elefsinioti A, Torti F, Krueger J, Rybak A, Maier L, Mackowiak SD, Gregersen LH, Munschauer M, et al: Circular RNAs are a large class of animal RNAs with regulatory potency. Nature 495: 333-338, 2013.

11. You X, Vlatkovic I, Babic A, Will T, Epstein I, Tushev G, Akbalik G, Wang M, Glock C, Quedenau C, et al: Neural circular RNAs are derived from synaptic genes and regulated by development and plasticity. Nat Neurosci 18: 603-610, 2015.

12. Su M, Xiao Y, Ma J, Tang Y, Tian B, Zhang Y, Li X, Wu Z, Yang D, Zhou Y, et al: Circular RNAs in cancer: Emerging functions in hallmarks, stemness, resistance and roles as potential biomarkers. Mol Cancer 18: 90, 2019.

13. Sun H, Xi P, Sun Z, Wang Q, Zhu B, Zhou J, Jin H, Zheng W, Tang W, Cao $\mathrm{H}$ and Cao X: Circ-SFMBT2 promotes the proliferation of gastric cancer cells through sponging miR-182-5p to enhance CREB1 expression. Cancer Manag Res 16: 5725-5734, 2018.

14. Zhang Y, Liu H, Li W, Yu J, Li J, Shen Z, Ye G, Qi X and Li G: CircRNA_100269 is downregulated in gastric cancer and suppresses tumor cell growth by targeting miR-630. Aging (Albany NY) 9: 1585-1593, 2017.

15. Iqbal $\mathrm{N}$ and Iqbal $\mathrm{N}$ : Human epidermal growth factor receptor 2 (HER2) in cancers: Overexpression and therapeutic implications. Mol Biol Int 2014: 852748, 2014

16. Yan M, Schwaederle M, Arguello D, Millis SZ, Gatalica Z and Kurzrock R: HER2 expression status in diverse cancers: Review of results from 37,992 patients. Cancer Metastasis Rev 34: 157-164, 2015.

17. Abrahao-Machado LF and Scapulatempo-Neto C: HER2 testing in gastric cancer: An update. World J Gastroenterol 22: 4619-4625, 2016.

18. Livak KJ and Schmittgen TD: Analysis of relative gene expression data using real-time quantitative PCR and the 2(-Delta Delta C(T)) method. Methods 25: 402-408, 2001.

19. $\mathrm{Hu} \mathrm{J}$ and $\mathrm{He} \mathrm{X}$ : Enhanced quantile normalization of microarray data to reduce loss of information in gene expression profiles. Biometrics 63: 50-59, 2007.

20. Yang Y, Wen L and Zhu H: Unveiling the hidden function of long non-coding RNA by identifying its major partner-protein. Cell Biosci 5: 59, 2015.

21. Lei M, Zheng G, Ning Q, Zheng J and Dong D: Translation and functional roles of circular RNAs in human caner. Mol Cancer 19: 30, 2020.

22. Qiu L, Wang T, Ge Q, Xu H, Wu Y, Tang Q and Chen K: Circular RNA signature in hepatocellular carcinoma. J Cancer 10: 3361-3372, 2019.

23. Lei B, Tian Z, Fan W and Ni B: Circular RNA: A novel biomarker and therapeutic target for human cancers. Int J Med Sci 16: 292-301, 2019.

24. Vo J, Cieslik M, Zhang Y, Shukla S, Xiao L, Zhang Y, Wu YM, Dhanasekaran SM, Engelke CG, Cao X, et al: The landscape of circular RNA in cancer. Cell 176: 869-881, 2019.

25. Kristensen LS, Hansen TB, Venø MT and Kjems J: Circular RNAs in cancer: Opportunities and challenges in the field. Oncogene 37: 555-565, 2018.

26. Fang X, Wen J, Sun M, Yuan Y and Xu Q: CircRNAs and its relationship with gastric cancer. J Cancer 10: 6105-6113, 2019.

27. Shi P, Wan J, Song H and Ding X: The emerging role of circular RNAs in gastric cancer. Am J Cancer Res 8: 1919-1932, 2018.

28. Zhu Z, Rong Z, Luo Z, Yu Z, Zhang J, Qiu Z and Huang C: Circular RNA circNHSL1 promotes gastric cancer progression through the miR-1306-3p/SIX1/vimentin axis. Mol Cancer 18: 126, 2019.

29. Perron G, Jandaghi P, Solanki S, Safisamghabadi M, Storoz C, Karimzadeh M, Papadakis AI, Arseneault M, Scelo G, Banks RE, et al: A general framework for interrogation of mRNA stability programs identifies RNA-binding proteins that govern cancer transcriptomes. Cell Rep 23: 1629-1650, 2018.

30. Benjamin D and Moroni C: mRNA stability and cancer: An emerging link? Expert Opin Biol Ther 7: 1515-1529, 2007.

31. Sun X, Hu Y, Wu J, Shi L, Zhu L, Xi PW, Wei JF and Ding Q: RBMS2 inhibits the proliferation by stabilizing P21 mRNA in breast cancer. J Exp Clin Cancer Res 37: 298, 2018. International (CC BY-NC-ND 4.0) License. 